\title{
PULMONARY MANIFESTATIONS OF STAPHYLOCOCCAL PYAEMIA
}

\author{
BY \\ D. R. HAY \\ From Christchurch Hospital, Christchurch, New Zealand
}

(RECEIVED FOR PUBLICATION SEPTEMBER 30, 1959)

In a previous study (Hay, 1960), the incidence of staphylococcal septicaemia and pyaemia at the Christchurch Hospital was shown to have risen considerably in recent years. The patients presented in a variety of ways, and frequently pulmonary signs or a chest radiograph gave the clue to the diagnosis. The object of this paper is to review the natural history of staphylococcal pyaemia as it affects the lungs (hereafter called staphylococcal pyaemic pulmonary disease).

\section{Patients Studied}

Seventy-one patients in the years 1957 and 1958 were proved by blood culture or necropsy to have staphylococcal septicaemia or pyaemia. Thirtyone of these were judged to have pulmonary complications, and, together with two other patients seen during January, 1959, form the basis of this study. The criteria for inclusion in the pulmonary group were radiological abnormalities appearing during the acute illness, or signs of metastatic suppurative infarcts in the lungs at necropsy.

The diagnosis of staphylococcal septicaemia was suspected during life in 29 of the 33 patients, and confirmed by blood cultures in 23 of these. In the remainder the diagnosis was confirmed at necropsy. Pyaemic pulmonary complications were suspected or confirmed during life in 26 patients; the seven other cases were revealed by necropsy.

Age and Sex Incidence and Mortality.-The 33 patients included 20 men and 13 women. Their ages ranged from 2 days to 79 years, with a mean of 27 years. Fifteen patients $(45 \%)$ died.

UNDERLYING DisEASES.-The diseases underlying the staphylococcal septicaemia were the following :

Post-operative septicaemia $\quad \ldots \quad \ldots \quad 9$ patients Infections of the head and neck $\ldots .99 \%$ Osteomyelitis and suppurative arthritis 9 " Umbilical infections $\quad \ldots \quad \ldots \quad \ldots 3^{\prime}$,

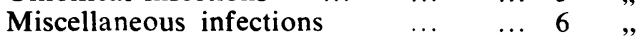

Clinical features of Staphylococcal Pyaemic Pulmonary Disease

This analysis of symptoms and signs is based on the findings in the 26 patients with staphylococcal pyaemic pulmonary disease diagnosed during life.

Presenting Symptoms.-Chest pain was the most frequent symptom and occurred in 12 out of 21 patients over the age of 5 years. The pain was usually pleural in character, but was associated with a friction rub in only four instances. It was never of marked severity and lasted one to three days. Cough occurred in six patients and was thus infrequent as a presenting or late symptom. Only three patients produced purulent sputum at the time of diagnosis; one patient coughed up blood-stained sputum, and five complained of dyspnoea.

Presenting Pulmonary Physical Signs.-The most frequent presenting physical signs of staphylococcal pyaemic pulmonary disease are listed in Table I. Physical signs were present in

$$
\text { TABLE I }
$$

PRESENTING PULMONARY SIGNS IN 26 PATIENTS WITH STAPHYLOCOCCAL PYAEMIC PULMONARY DISEASE DIAGNOSED DURING LIFE

\begin{tabular}{|c|c|c|c|c|c|}
\hline \multicolumn{5}{|c|}{ Pulmonary Signs } & $\frac{\text { No. of Cases }}{8}$ \\
\hline $\begin{array}{l}\text { Scattered rales in o } \\
\text { Increased respirato } \\
\text { Consolidation } \\
\text { Pleural friction rub } \\
\text { Shock } \\
\text { Pneumothorax } \\
\text { Pleural fluid } \\
\text { No physical signs }\end{array}$ & 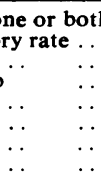 & $\begin{array}{l}\text { ngs } \\
\cdots \\
\cdots \\
\cdots \\
\cdots \\
\cdots \\
\cdots\end{array}$ & $\begin{array}{l}\cdots \\
\cdots \\
\cdots \\
\cdots \\
\cdots \\
\cdots\end{array}$ & $\begin{array}{l}\cdots \\
\cdots \\
\cdots \\
\cdots \\
\cdots \\
\cdots \\
\cdots\end{array}$ & $\begin{array}{r}8 \\
11 \\
6 \\
4 \\
3 \\
1 \\
2 \\
5\end{array}$ \\
\hline
\end{tabular}

most patients, but they were never gross and consisted usually of an increase in the respiratory rate or an alteration in the character of breathing, together with scattered moist sounds in one or both lungs. Obvious consolidation with bronchial breathing was unusual, and pleural friction was much less frequent than pleural pain. The initial radiological changes were usually inconspicuous, and this is reflected in the paucity of physical 
signs. Three patients showed signs of shock, together with fever and respiratory distress. These included two post-operative patients, one of whom also had an A-V nodal tachycardia, and a woman with an overwhelming infection which was believed to arise from the pelvis.

Later Pulmonary Symptoms and Signs.-Few of the patients developed outstanding pulmonary symptoms, and widespread radiological changes were often present without cough or sputum, and with only minor pulmonary physical signs. Adventitious sounds occurred frequently and were often bilateral, and disappeared after seven to 10 days. The most striking symptoms and signs developed when a pneumothorax complicated the illness. This occurred in four patients, two of whom were infants with fatal umbilical infection. The third patient (Case 31) had osteomyelitis of the clavicle and showed signs of bilateral consolidation on admission. A tension pneumothorax developed on the right side on the third day, and two days later a pneumothorax occurred on the left side. After a stormy illness he slowly recovered, but it was not until weeks later, when a pathological fracture appeared in the right clavicle, that the original site of the staphylococcal infection was revealed. The pneumothorax in the fourth patient (Case 72) was revealed by a routine radiograph taken 21 days after the onset of pulmonary disease. He had not complained of dyspnoea but had physical signs of a pneumothorax. In both the previous patients, the pneumothorax occurred first in the lung showing the smaller amount of pulmonary consolidation ; both patients recovered well with intercostal drainage.

It was not uncommon for a trace of fluid to be demonstrated radiologically in one or both pleurae. This occurred in 11 patients, in five of whom the fluid was bilateral, but in only two was there sufficient to be obvious or significant clinically. Empyema occurred once only, in an infant with fatal parotitis and facial infection.

There were few patients who produced sputum, and haemoptysis never occurred as a late symptom. The change from solid pulmonary lesions to small cavities was not accompanied by a productive cough.

The symptoms and signs of the underlying primary staphylococcal illness often dominated the clinical picture, and identification of the causative organism was readily possible from culture of various purulent discharges. The details of the primary illnesses will not be further considered in this paper.

\section{Radiological Signs of Staphylococcal Pyaemic Pulmonary Disease}

Relation of Radiological AbNormalities to Time of Positive Blood Culture.-In 16 of the 23 patients with positive blood cultures, the first chest radiograph taken was abnormal. In eight the abnormality was present before the blood culture was taken, the diagnosis of staphylococcal pyaemia being suggested by the radiograph. The other eight patients all had radiographic abnormalities on the same day as the positive blood culture. In five other patients a radiograph was taken within a week of the positive culture and found to be abnormal.

In one patient the radiograph on the day of the positive blood culture was normal, but patchy consolidation in the left lower lobe was demonstrated in a radiograph two weeks later.

Another infant in whom the diagnosis was not confirmed by blood culture had a normal radiograph one day, followed by a spontaneous pneumothorax the next. Another child of 8 (Case 39) had a normal radiograph two days after a positive blood culture, but at necropsy five days later there were scattered small metastatic pulmonary abscesses. In two other patients, normal chest radiographs were reported after the diagnosis of staphylococcal pyaemia had been made, but necropsy revealed pulmonary lesions. One was a woman of 62 years (Case 41) with suppurative arthritis of the shoulder, who had a normal radiograph seven days after a positive blood culture, but was shown to have a large septic infarct in the right middle lobe at necropsy a week later.

Early Radiological Signs. - The earliest radiological signs of staphylococcal pyaemic pulmonary disease consist of oval or round, low density shadows situated peripherally, usually in the middle zone of the lungs. The diameter of the opacities may range from 0.5 to $1.5 \mathrm{~cm}$., and only one may be visible in the first radiograph. Interpretation may be difficult when such solitary lesions are present, but almost invariably second films taken within one to four days will reveal further lesions or a slight increase in the size of the shadows. In the presence of a history suggestive of staphylococcal septicaemia, these minor lesions are most significant and may point to the correct diagnosis. This is well illustrated by Case 71 in which the original diagnosis was rheumatic fever. An urgent chest radiograph showed a $1 \mathrm{~cm}$. opacity in the left middle zone which increased in size within 48 hours, when 


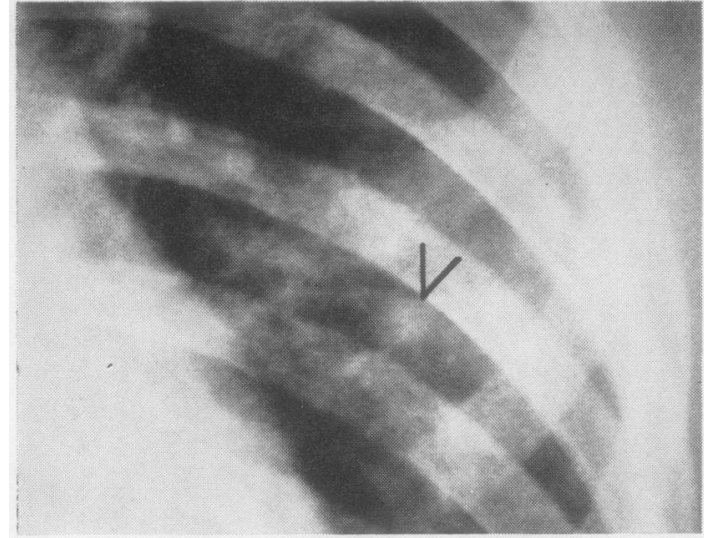

Fig. 1.-Case No. 71: Close-up view of the left mid-zone to show early pyaemic lung lesions in a patient with acute osteomyelitis.

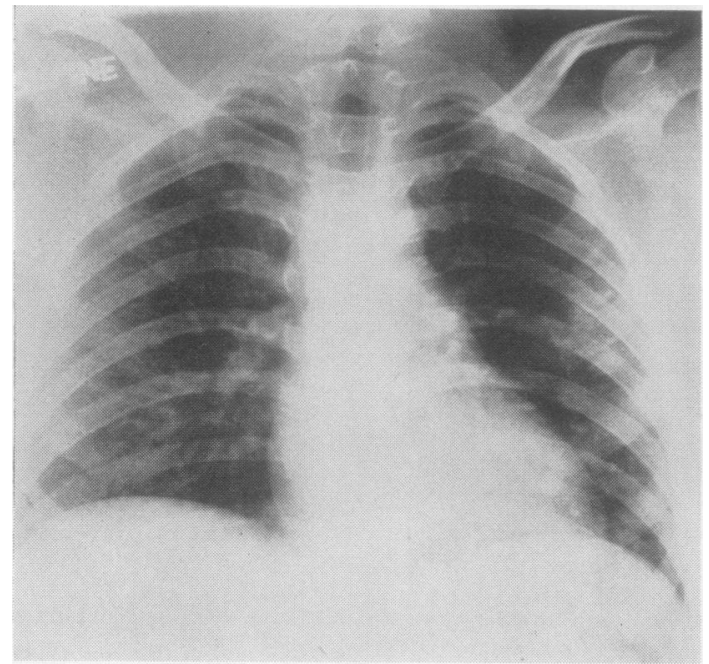

Fig. 3.-Case No. 27: Early pyaemic lesions in the right and left mid-zones in the admission radiograph of a boy with fulminating osteomyelitis.

other shadows had also appeared (Figs. 1 and 2). Subsequently he developed a pneumothorax. Another boy, who died from fulminating infection arising from osteomyelitis (Case 27), also showed unimpressive small bilateral opacities in the radiograph taken on admission, but within 24 hours the extent of consolidation had increased considerably (Figs. 3 and 4).

Pleural effusion was rarely seen as an early sign, and on only two occasions was a pneumothorax present within two days of diagnosis.

Later Radiological Signs.-In 11 surviving patients whose early radiographs showed typical pyaemic lesions, frequent subsequent films were

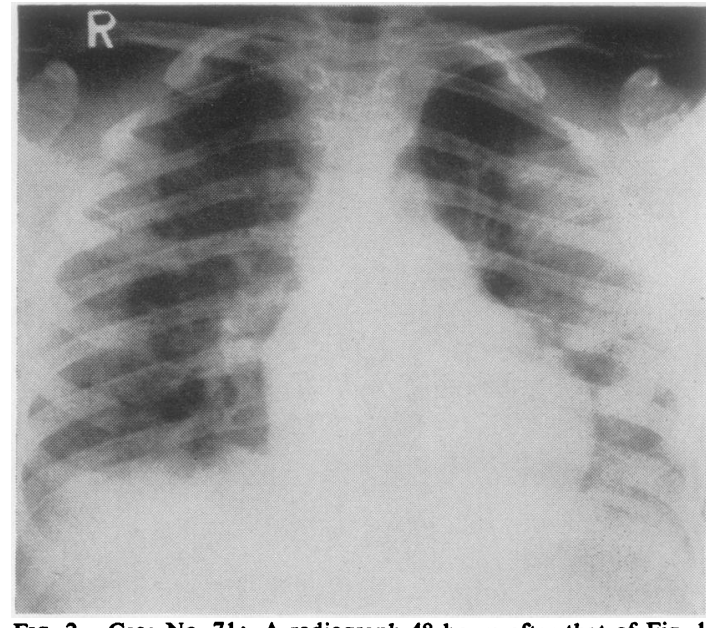

Fig. 2.-Case No. 71: A radiograph 48 hours after that of Fig. 1, showing scattered, ill-defined pyaemic lesions in both lungs.

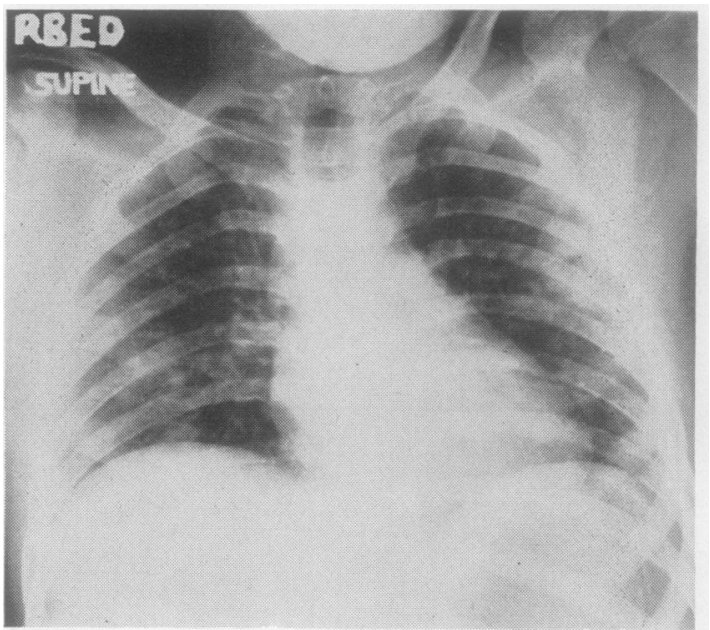

FIG. 4.-Case No. 27: The same patient as in Fig. 3, showing increased pulmonary consolidation within 24 hours.

taken in order to follow the natural history of this complication of staphylococcal infection. The pulmonary consolidation was most marked on the first radiograph in four patients, but in the remainder the most advanced consolidation was not evident until the fifth day. In all patients treatment with appropriate antibiotics had been instituted from the time of the earliest radiograph or before.

The size of the pyaemic infarcts was variable, averaging about 1.5 by $1.0 \mathrm{~cm}$. but at times as large as 4.5 by $6.0 \mathrm{~cm}$. Progression of the pulmonary disease was more often revealed by the appearance of new lesions than by the increase 


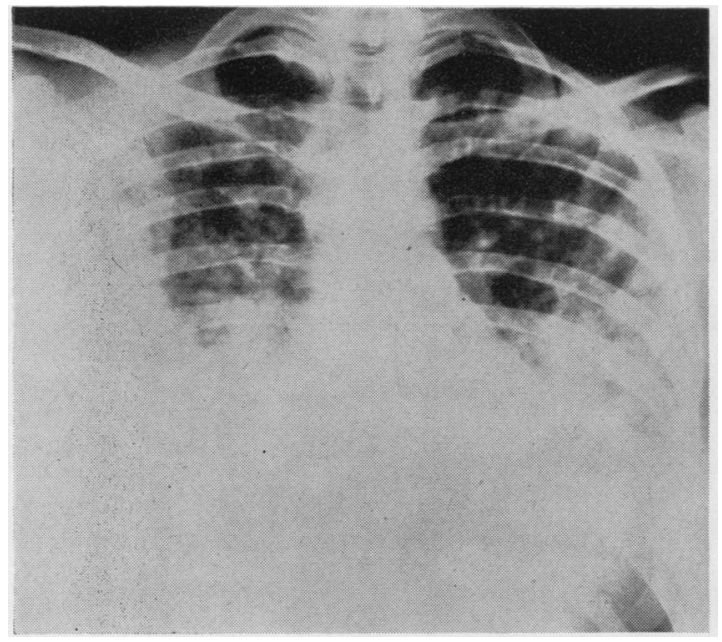

FIG. 5.-Case No. 63: Very extensive bilateral consolidation in a patient with fulminating staphylococcal pyaemia.

in size of existing ones. In occasional patients, the infarcts were more confluent, as in Case 63 of which the radiograph is illustrated in Fig. 5. At necropsy, the suppurative foci were shown to be based around blood vessels containing septic thrombi, which appeared to have come from an area of suppurative thrombophlebitis in the pelvis.

In nine of the 11 patients under analysis and in two others of the series, the development of small "cavities" or cysts was noted. These were round or oval, thin-walled, air-containing lesions, about 1.5 to $1 \mathrm{~cm}$. in diameter, situated at the periphery of the lung, in close relation to the pyaemic infarcts. Fluid levels were rarely seen and ultimately these "ring shadows" disappeared with almost no residual abnormality. In two patients, the cavities were present in the first radiograph, but for the remainder the average time of appearance was on the seventh day. More than one was usually present at a time and sometimes as many as six. Typical examples are shown in Figs. 6 and 7.

These lesions are thought not to be necrotic cavities, but to develop as the result of regional obstructive emphysema secondary to the acute inflammatory process around the pyaemic infarcts. Sometimes the small cysts may coalesce to form large bullae, and either small or large lesions may rupture and cause a pneumothorax. This complication occurred in two patients of the group, and is illustrated in Fig. 8.

Pleural effusion developed later in 11 patients, but was usually only a trace at the costophrenic angle, except in two instances when the quantity

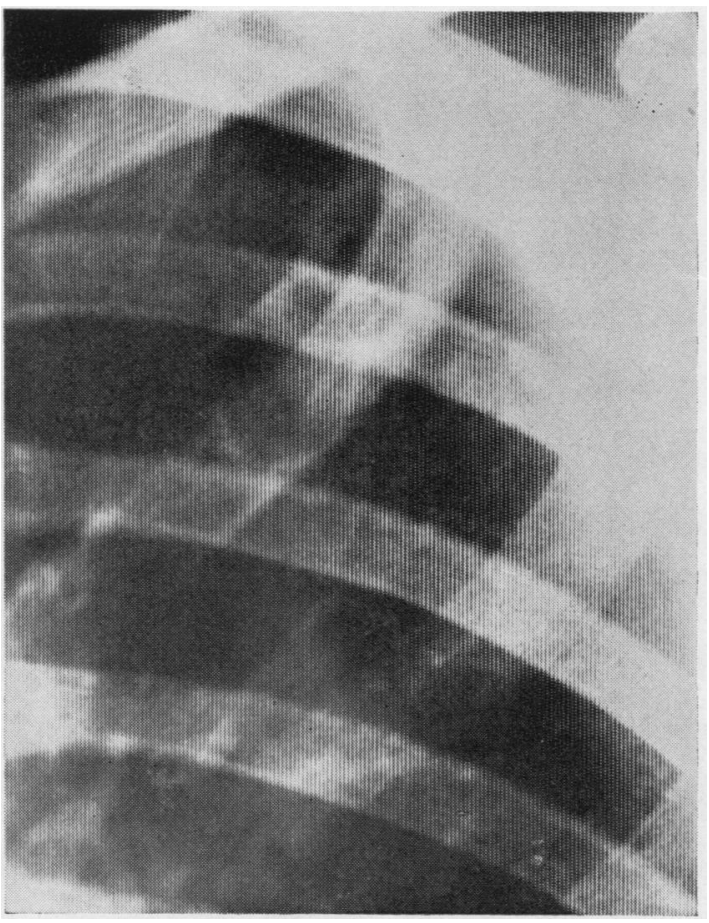

Fig. 6.-Case No. 48: Close-up view of a typical "cavity" or cyst in the left upper lobe.

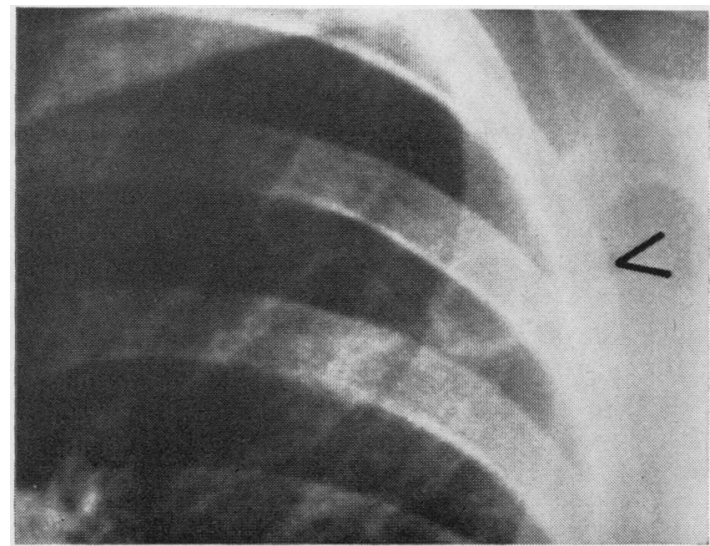

FIG. 7.-Case No. 47: Thin-walled " cavity" in the left upper lobe of a patient with pyaemia following a facial boil.

was moderate. Small bilateral effusions occurred in five patients.

Ten patients with staphylococcal pyaemic pulmonary disease, reviewed later, have shown almost normal chest radiographs two to three months after the acute illness. Further examples of the radiological abnormalities are shown in Figs. 9 and 10. 


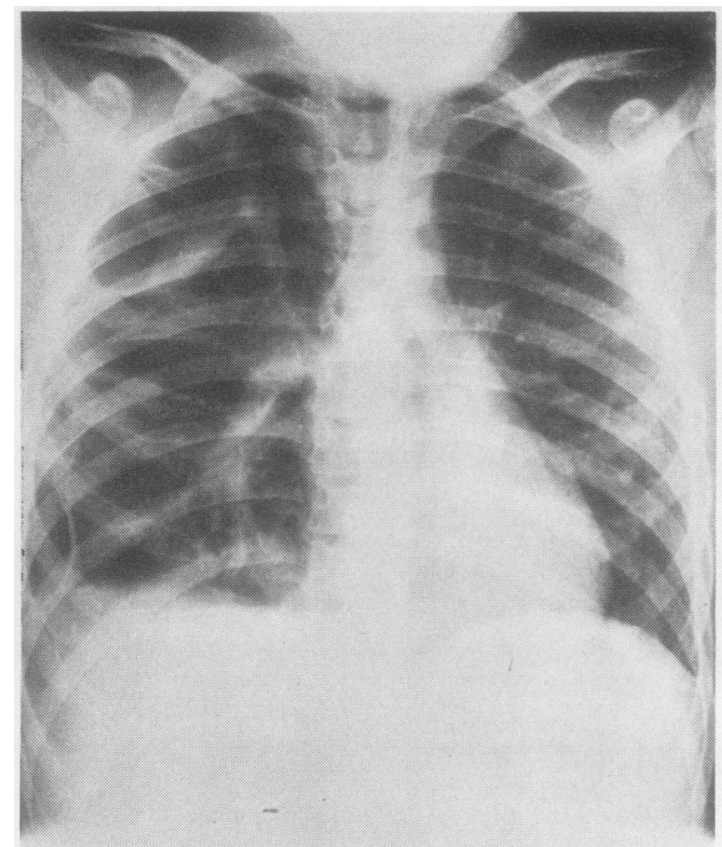

Fig. 8.-Case No. 71: A large bulla in the right mid-zone, and a pneumothorax with adhesions on the same side. Small cysts are also present in the left mid-zone.

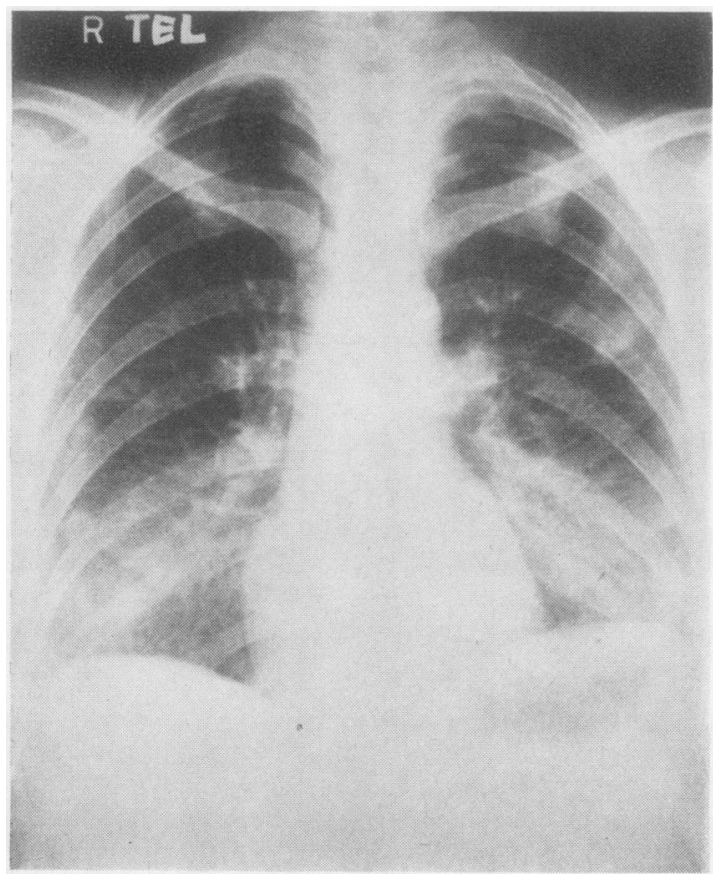

FIG. 10.-Case No. 40: The same patient as in Fig. 9 showing considerable resolution within two weeks. A subsequent radiograph showed no residual abnormality.

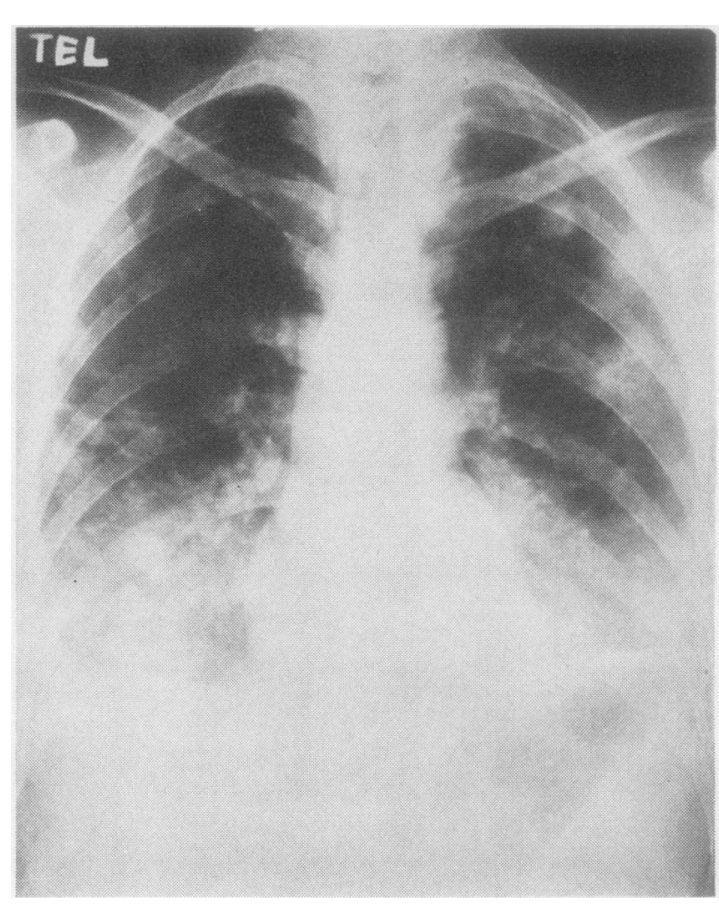

FIG. 9.-Case No. 40: Scattered pyaemic infarcts in the periphery of both lungs in a patient with pyaemia secondary to facial infection. There is a trace of fluid in both pleural cavities.

\section{BACTERIOLOGY}

As most patients produced no sputum. it was difficult to obtain specimens for bacteriological examination. Sputa from 12 patients were examined and nine grew staphylococci, the antibiotic sensitivities of which paralleled the results 3 . from blood cultures. In 10 other patients, swabs $\delta$ taken from the lung at necropsy grew staphylo- 3 cocci which were penicillin-resistant in eight 0 instances. Of the 33 patients in the pulmonary series, only six $(18 \%)$ were infected by penicillinsensitive staphylococci. This is somewhat lower than the figure of $28 \%$ for the larger series of $\widetilde{N}$ staphylococcal septicaemia (Hay, 1960). However. N it would be unjustified to infer from this figure N that infections by penicillin-resistant organisms were more likely to be complicated by pyaemic pulmonary disease.

\section{Pathology}

Necropsies were performed in all 15 fatal $\stackrel{0}{0}^{\circ}$ cases. A fibrinous exudate was frequently $\underset{\mathbb{D}}{\mathbb{D}}$ present on the pleural surface of the lungs, with $\mathbb{\mathbb { D }}$ small effusions in several cases but an empyema in only one. The metastatic abscesses were usually visible to the naked eye and were characteristic- 
ally subpleural in situation and unrelated to major bronchi. In the early stages, these lesions consisted of discrete areas of haemorrhagic consolidation, the centre of which later became necrotic and pale and finally obviously purulent. Small air-filled cysts in relation to the abscesses were noted in several patients, but never were there large chronic abscesses. On microscopic examination, the foci of inflammation and suppuration were shown to be related to blood vessels and septic clot was frequently demonstrated in both arteries and veins. Colonies of darkly-staining staphylococci were obvious in the sections.

\section{Treatment}

The antibiotic treatment of the patients in this study has been discussed elsewhere. Drugs which were considered appropriate from the results of sensitivity tests in vitro were given in high dosage and continued for a month. The response to treatment was related to the nature of the underlying staphylococcal illness, its duration, and the delay before diagnosis was made. Three patients died within 48 hours of admission, presumably from the effect of overwhelming toxaemia. The successes in therapy were achieved by prolonged and adequate treatment with penicillin when indicated or with combinations of erythromycin and novobiocin. Surgical treatment was required only for the management of spontaneous pneumothoraces. Postural drainage and other forms of physiotherapy were of no value in these patients.

\section{Discussion}

The high incidence of serious staphylococcal disease has been one of the notable features of medical practice at the Christchurch Hospital in the last three years. Staphylococcal septicaemia must now enter into the differential diagnosis of any unexplained pyrexia or post-operative collapse. When there are obvious foci of staphylococcal infection, more serious complications are possible and should be excluded by urgent blood cultures.

In the present study $44 \%$ of patients with staphylococcal septicaemia had pulmonary complications, and characteristic radiographic changes were frequent. Chest radiographs are desirable early, whenever this diagnosis is suspected, and should be repeated within two days if the first radiograph shows suspicious lesions. The diagnostic value of apparently trivial pulmonary lesions in confirming suspected staphylococcal pyaemia was demonstrated repeatedly, and in a number of patients the diagnosis was first suggested by the radiologist. Although most patients with staphylococcal pyaemic pulmonary disease showed radiological abnormalities at some time, in a few the lesions were too small to produce a visible opacity. Thus the incidence of pulmonary miliary abscesses at necropsy exceeded the number demonstrated during life. The size of the abscesses and the delay before they became radiologically visible are doubtless related to the virulence of the organism and the resistance of the host.

Pulmonary physical signs were usually present when metastatic pulmonary complications had occurred, but symptoms were less common. Chest pain was the most important, and its frequency was related to the subpleural situation of many of the pulmonary infarcts. The important physical signs were an increased respiratory rate and adventitious sounds in one or both lungs. These abnormalities were often trivial and required careful observation for their detection.

The prognosis of staphylococcal pyaemic pulmonary disease is difficult to assess, for it is the underlying primary staphylococcal infection which often determines the patient's fate. The mortality in this particular group was $45 \%$, which is slightly less than the figure of $50 \%$ for the larger series of patients with staphylococcal septicaemia (Hay, 1960). In the patients who recovered, extensive bilateral consolidation often resolved completely and the small cavities or cysts which were conspicuous during the acute illness disappeared entirely. Follow-up bronchograms were not undertaken, but in view of the normal radiographs taken after three months it seems unlikely that these patients were left with significant structural damage to the lungs. This is in contrast to primary staphylococcal pneumonia, which may lead to the development of bronchiectatic changes in the affected lung segments.

Except for the one patient whose radiograph is illustrated in Fig. 5, the pulmonary disease itself was probably never the primary cause of death. Pyaemic spread to the lungs always leads to the danger of pneumothorax, for which careful clinical and radiological watch must be kept. Empyema, on the other hand, was not a problem in these patients, which is surprising in view of the nature and situation of the suppurative infarcts. Perhaps the antibiotic treatment prevented this complication, although it did not always prevent an increase in the extent of pulmonary consolidation.

The frequency of pulmonary involvement in published accounts of staphylococcal septicaemia 
is difficult to assess, and there is little recent information on the subject. Mendell (1939) found a $63 \%$ incidence of pulmonary complications in 35 patients with staphylococcal septicaemia, while Skinner and Keefer (1941) observed metastatic respiratory tract infections in $30 \%$ of 122 patients. In a more recent report, Schirger, Martin, and Nichols (1957) record only three cases in 109 antibiotic-treated patients with staphylococcal bacteraemia, while Hassall and Rountree (1959) make no mention of pulmonary complications among their 86 patients with staphylococcal septicaemia.

Primary staphylococcal pneumonia or pneumonia complicating influenza or other virus infections of the respiratory tract is common (Oswald, Shooter, and Curwen, 1958 ; Robertson, Caley, and Moore, 1958), but this disease should be distinguished from pyaemic staphylococcal infection of the lungs. The former is characterized by lobar or multilobar consolidation, and by conspicuous respiratory symptoms and signs. Sputum is usual, empyema is common, but positive blood cultures are seldom observed. In staphylococcal pyaemic pulmonary disease, the signs of the underlying infection often predominate and the respiratory signs may well be overlooked. The chest radiograph shows characteristic multiple small opacities, and blood cultures are almost always positive. Cavitation and pneumothoraces may occur in either condition, but persistent structural damage is unusual in pyaemic infection. Successful treatment demands the use of appropriate antibiotics for periods of three to four weeks in the case of pyaemic pulmonary disease, but results remain somewhat unsatisfactory.

\section{SUMMARY}

Thirty-three patients with pulmonary complications of staphylococcal pyaemia have been studied.
Pulmonary symptoms and signs were inconspicuous : chest pain was the most common symptom, and an increased respiratory rate and scattered moist sounds the most important physical signs.

The characteristic radiological signs were multiple, oval, or round opacities situated in the $\vec{\circ}$ periphery of both lungs. Thin-walled cavities or cysts developed in 11 patients, but almost complete resolution eventually occurred. Four patients developed pneumothoraces, and in 11 there $\vec{x}$ were small pleural effusions. Repeated chest $\vec{G}$ radiographs were valuable in assessing suspicious lesions.

Only six patients (18\%) were infected by penicillin-sensitive staphylococci. Staphylococci were grown from the sputum of nine of the 12 patients who produced specimens.

Fifteen patients $(45 \%)$ died, but in only one was $\stackrel{\frac{\partial}{丂}}{0}$ the pulmonary disease the main cause of death. Successful treatment demanded prolonged courses of antibiotics.

The incidence of pulmonary complications was $44 \%$ of a series of 71 patients with staphylococcal septicaemia, a figure higher than in other recently published series.

I wish to thank the physicians and surgeons of the $\overrightarrow{\overrightarrow{0}}$ Christchurch Hospital for permission to study their 3 patients. I am grateful to Dr. G. L. Rolleston and $\rightleftharpoons$ to my wife, Dr. Jocelyn Hay, for their advice ando criticism, and to Mrs. Margaret Meyer for her secretarial assistance. The illustrations were prepared by Messrs. K. Donaldson and S. Brooks.

\section{REFERENCES}

Hassall, J. E., and Rountree, P. M. (1959). Lancet, 1, 213.

Hay, D. R. (1960). Ouart. J. Med. In the press.

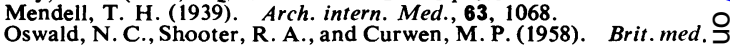

Robertson, L., Caley, J. P., and Moore, J. (1958). Lancet, 2, 233. Schirger, A., Martin, W. G., and Nichols, D. R. (1957). Ann. intern. Skinner, D., and Keefer, C. S. (1941). Arch.intern. Med., 68, 851. N 